\title{
Are obese adolescents more prone to get infections? The HELENA study
}

\author{
L. E. Díaz ${ }^{1}$, S. Gomez-Martínez ${ }^{1}$, J. Warnberg ${ }^{2}$, L. Moreno ${ }^{3}$, J. R. Ruiz ${ }^{4}$, K. Widhalm ${ }^{5}$, A. Kafatos ${ }^{6}$, \\ C. Breidenassel ${ }^{7}$, M. Ferrari ${ }^{8}$, J. Repassy ${ }^{9}$, I. Huybrechts ${ }^{10}$ and A. Marcos
}

${ }^{1}$ Immunonutrition Research Group, Department of Metabolism and Nutrition, Institute of Food Science and Technology and Nutrition (ICTAN), Spanish National Research Council (CSIC), Madrid, Spain, ${ }^{2}$ Department of Preventive Medicine and Public Health, University of Navarra, Pamplona, Spain, ${ }^{3}$ College of Health Sciences, University of Zaragoza, ${ }^{4}$ Department of Physical Education and Sport, Faculty of Physical Activity and Sport Sciences, University of Granada, Granada, Spain, ${ }^{5}$ Department of Paediatrics, Division of Clinical Nutrition, Medical University of Vienna, ${ }^{6}$ Department of Social Medicine, Preventive Medicine and Nutrition Clinic, School of Medicine, University of Crete, Crete, Greece, ${ }^{7}$ Department of Nutrition and Food Sciences-Nutritional Physiology, University of Bonn, Bonn, Germany, ${ }^{8}$ National Research Institute on Food and Nutrition, Rome, Italy, ${ }^{9}$ Department of Paediatrics, University of Pécs, Pécs-József A.7, Pecs, Hungary and ${ }^{10}$ Department of Public Health, Ghent University, Ghent, Belgium

Obesity has been related not only to hypercholesterolaemia, hypertension, cardiovascular diseases and certain tumours but also to immune dysfunction, which is accompanied by a high rate of infections. Studies in obese human subjects and animals have reported increased adiposity affecting the humoral immune response, which is essential to block host pathogen invasion. Therefore, this study was aimed at determining possible association between some humoral immune markers and body composition in healthy adolescents from the HELENA cross-sectional study.

A subsample of 1000 healthy adolescents ranged between 12.5 and 17.5 years old was selected. Blood samples were analysed for leucocyte, B-lymphocyte and serum Ig levels. CD19 (B-cells) subset was analysed by flow cytometry (FACSCAN PLUS DUAL LASER, Becton Dickinson Sunnyvale, CA, USA). Serum IgG, IgA and IgM concentrations were determined by nephelometry. Height, weight, waist and hip circumference values were measured according to the methodology described elsewhere ${ }^{(1)}$. BMI was calculated.

In the obese group (boys and girls), only IgA showed significant differences in boys in comparison with optimum BMI and overweight $(176 \pm 90.2$ v. $134 \pm 61.2$ and $138 \pm 59.9$, respectively). Positive correlations were also found between IgA with waist $(r=0.110$, $P=0.018)$ and hip $(r=0.123, P=0.008)$ circumferences.

These results showed that serum IgA concentration was the most sensitive marker, probably suggesting a higher risk of infection in obese boys than in the rest of the groups.

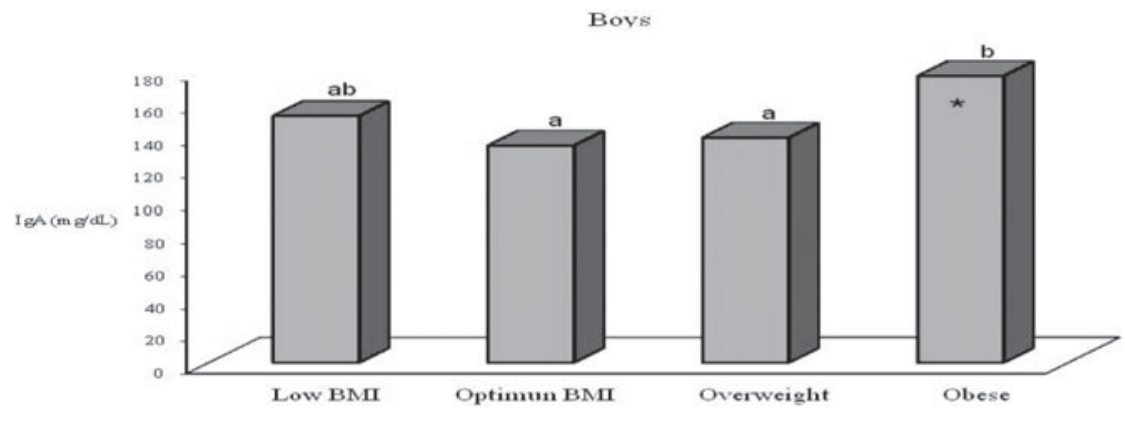

a,b, Significant differences between groups. Student's $t$ test $(P<0.05)$, *Positive correlations between $\operatorname{IgA}$ with waist $(r=0.110$, $P=0.018)$ and hip $(r=0.123, P=0.008)$ circumferences.

1. Nagy E, Vicente-Rodriguez G, Manios Y, et al. (2008) Int J Obes 32, S58-S65. 